\title{
Acupuncture Efficacy Study Using Electromyographic Signals
}

\author{
Yassine Sabri, Siham Lamzabi, Aouad Siham, Aberrahim Maizate \\ Laboratory of Innovation in Management and Engineering for Enterprise (LIMIE), \\ ISGA Rabat,27 ,Address, Zip Code \\ Avenue Oqba, Agdal Rabat, Morocco \\ Mohammed V University of Rabat ,Smart Systems Laboratory (SSL) ENSIAS,Morocco \\ Ritm-ESTC/CEDENSE, Hassan II university km7, Route d'El Jadida, Casablanca Morocco
}

Received: April 23, 2021. Received: September 10, 2021. Accepted: October 10, 2021. Published: October 18, 2021..

\begin{abstract}
Acupuncture is a centuries-old therapeutic technique. However, because of the large number of complicating circumstances, it has been difficult to clearly prove the treatment's therapeutic effectiveness.As a result, acupuncture has failed to acquire acceptance in the mainstream clinical sector. An electromyography (EMG) sensor was built and used to test the efficacy of acupuncture in alleviating muscular stiffness in this study. Electrodes, differential and inverting amplifiers, filters, and a full-wave rectifier made up the EMG circuit. The output of the circuit was sent to a microcontroller for analog-to-digital transformation in order to perform data acquisition. Acupuncture was used to treat four participants who had muscular dysfunction in various regions of their bodies in our case study. Before and after the therapy, EMG signals at the damaged regions were recorded. The findings revealed that the therapy had no immediate conceivable impact on the patients, since the levels of muscular contraction before and after the treatment were comparable. When the EMG signals were measured 30 minutes after the therapy, signs of muscular alleviation were found. This shows that acupuncture does supply patients with beneficial medicine, although slowly. The act of placing the highly conducting needles into the acupuncture sites, we believe, is similar to connecting a parallel wire to a circuit, resulting in a short-circuited route at the meridian. It permits the meridian's polarized inner energy, or qi, to pass through. The equilibrium in qi regulation can therefore be restored by unclogging the flow of qi.The repair process is relatively slow, and the treatment impact may not be immediately apparent, because the constitutive qualities at the acupuncture points where the needles are pricked may not alter quickly.
\end{abstract}

\section{Keywords-}

Signal processing, Circuits, Therapeutic technique, Arduino .

\section{INTRODUCTION}

A CUPUNCTURE is an ancient therapeutic modality which has been practiced in China since thousands of years ago. According to traditional Chinese medicine (TCM), a form of interior bodily energy, which has been vaguely interpreted as qi in the literature, is generated in the internal organs and systems [1-3]. The qi is carried by the breath or air and circulates throughout the entire body, forming intricate interwoven paths known as the meridian system or ching-lo [1]. Physically, the meridians are perceived to be made up of groups of orderly arranged electrically polarized water molecules, which form water clusters with permanent electric dipole moment [2]. Acupuncture treatment is merely one of the possible therapies which manipulate the qi as it circulates the meridians to achieve curative effects [1]. The other examples which are based on this method are the cupping and moxibustion therapies, and the tai-chi and qi-gong exercises [4].

Despite its popular practice over millennia, the acupuncture treatment fails to gain worldwide acceptance within the mainstream orthodox clinical feld [2]. Due to the plethora of confounding factors enshrouding this treatment, researchers have found it extremely difficult to definitively and convincingly verify the curative efficacy of the treatment [5]. In other words, a tangible and descriptive demonstrable mechanism is yet to be formulated to prove its scientific reality [1]. In fact, many believe that acupuncture may, at best, merely introduce psychogenetic effect to the patients. In [6], Tough examined the immediate effects of acupuncture on the electromyography (EMG) activity of the common wrist extensor muscles. Three experiments were carried out on 35 subjects - namely, genuine acupuncture, inappropriate acupuncture, and a no condition control. At the end of the study, he drew the conclusion that the acupuncture interventions did not 
register any meaningful change on the EMG activity. Farida et al. [7], on the other hand, showed that the therapy produced positive results. The authors examined the effect of acupuncture on the EMG activity from the bicep muscles. In the case study, two groups, with four subjects in each group, received stimulation from $5 \mathrm{~kg}$ load. The subjects in the first group were administered acupuncture while those in the second were not. The authors concluded that acupuncture therapy was capable of improving human stamina, particularly, on muscle exercises. Although both [6,7] attempted to correlate the effect of acupuncture with the EMG activity, it is apparent that their outcomes are in stark contrast. The actual curative efficacy of acupuncture therefore remains uncertain.

In order to probe further the efficacy of acupuncture therapy in treating muscle spasticity and flaccidity, we conducted an in-depth case study based on patients suffering from muscle impairment at different parts of the body. An EMG sensor was constructed from off-the-shelf components, and it was implemented to measure contraction of muscles before and after the treatment. In this paper, the process of constructing the EMG circuit is first described in detail. This is then followed by the description of the case study. Analysis on the results and hypothesis on the underlying operational principal of acupuncture are discussed at the later sections of the paper.

\section{ELECTROMYOGRAPHY SENSOR}

Electromyography (EMG) sensors are used to detect and examine the electrical signals generated by the movement of muscles or stimulation of nerve to the muscles. The electrical signal detected by the sensor during the neuromuscular activities is known as the electromyographic (EMG) signal. The contraction and relaxation of muscles are stimulated by impulses in the neurons to the muscle. The EMG signal can therefore be expressed in terms of a train of Motor Action Unit Potentials (MUAPs) which shows the muscle response to neural stimulation [8]. Mathematically, the EMG signal can be written as [8].

$$
x(n)=\sum_{r=0}^{N-1} h(r) e(n-r)+w(n)
$$

where $\mathrm{x}(\mathrm{n})$ is the modeled EMG signal, $e(n)$ the point processed which represents the fring impulse, $h(r)$ the MUAPs, $w(n)$ the zero mean additive white Gaussian noise, and $\mathrm{N}$ denotes the number of motor unit fringes. The electrodes of an EMG sensor can be used to detect the ionic flow across the muscle membranes that propagate through the tissues in order to generate the EMG signal [9]. Hence, the sensor can be used to measure the anatomical and physiological status of the muscles. Traditionally, EMG signals are used in biomedical science for identifying neuromuscular disorders.
Nowadays, however, the applications of EMG signals have been expanded into the realm of biomedical engineering - it is also used for constructing EMGbased prosthetic, robotic and other useful systems. According to [8], the quality of the signal detected by the EMG sensor is highly affected by two factors - the first is the signal-to-noise ratio (SNR), whereas the second is the distortion of the signal. The SNR is used to measure the magnitude of the EMG signals in comparison to the that of the noise. When detecting the EMG signal, electrical noise is very often coupled along. The electrical noise includes the inherent noise in electronic equipment, ambient noise, motion artefact, and the inherent instability of the EMG signal [8]. The EMG signal is picked up by electrodes. It then undergoes a series of amplifications, filtering, and rectification. At each process step, the signal may lose energy at different rates. Hence, beside noise contamination, the signal may also face the risk of being distorted. To put it simply, the following conditions have to be observed in order to optimize the detection of the EMG signal:

- The magnitude of the EMG signal itself has to be optimal, while noise contamination has to be suppressed. This is to say, the higher is the SNR value, the better is the EMG signal detection.

- Signal distortion is to be minimized. Since EMG signal can be decomposed into a series of frequency-dependent components, the signal experiences distortion when each component attenuates differently. It is therefore important to ensure that the wave amplitudes of all frequency components sustain uniform losses. This is to say, the loss should be frequency-independent (or at least, close to independent), if not completely eliminated.

\section{A. MATERIALS AND COMPONENTS}

The EMG sensor used in our case study is based on the design in [10]. The components involved in the construction of the EMG circuit include three TL072 integrated circuits (ICs), an INA106 integrated circuit, three surface EMG electrodes, two $9 \mathrm{~V}$ batteries, two $1 \mu \mathrm{F}$ tantalum capacitors, a 0.01 and a $1 \mu \mathrm{F}$ ceramic capacitors, a $100 \mathrm{k} \Omega$ trimmer potentiometer, two $1 \mathrm{~N} 4148$ diodes and three $150 \mathrm{k} \Omega$, two $1 \mathrm{M} \Omega$, two $80.6 \mathrm{k} \Omega$, six $10 \mathrm{k} \Omega$ and one $1 \mathrm{k} \Omega$ resistors. A microcontroller is used to process the output signal, so that it could be recorded for further analysis. In this section, the functions of the important components shall be briefly elaborated.

According to Jamal [11], the contraction of muscle of human body can be detected by using different types of EMG electrodes. EMG electrodes 
can be either invasive or noninvasive. The invasive types are intramuscular electrodes and can be further divided into needle electrodes and fine wire electrodes. The noninvasive types are surface electrodes. In general, the invasive electrodes provide higher accuracy and sensitivity. The signals detected using the invasive electrodes are also more stable. However, since the process involved pricking the electrodes into the skin, most volunteers were against using them. Hence, we resort to the noninvasive surface electrodes in our work here. The surface EMG electrodes are electrodes applied onto the surface of the skin. Hence, they do not impose discomfort to the patients. In order to let the current to flow through the surface EMG electrodes, a chemical equilibrium between the sensing surface and the skin of the body will be formed by electrolytic conduction [11]. Surface electrodes are, nevertheless, relatively sensitive to electrical noise. One has to be extremely precautions when using the electrodes for EMG detection.

The INA106 IC is a differential amplifier that consists of a precision operational mplifier (op-amp) and four on-chip metal film resistors, i.e., two $10 \mathrm{k} \Omega$ resistors bnnected to the input pins and another two $100 \mathrm{k} \Omega$ resistors connected to the sense nd reference pins. The internal configuration of the chip is illustrated in Figure 1. NA106 produces accurate gain and relatively high common-mode rejection ratio CMRR) of $86 \mathrm{~dB}$ which enables it to reject the noise of input signal [12]. Hence, it employed at the first stage of the EMG circuit where the electrodes are connected irectly to the inputs. The TL072 IC consists of a low noise junction field effect transistor, JFET-input perational amplifier. The pin layout of the chip is depicted in paper Figure 2 As can be een from the figure, the chip consists of four inputs and two outputs, which means it

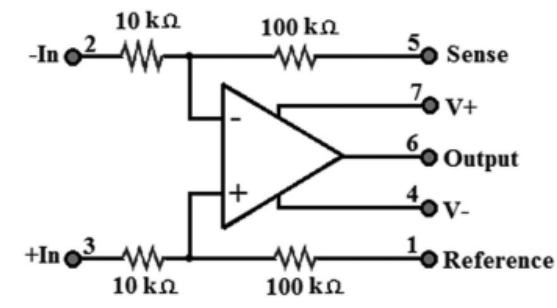

Fig. 1: Pin layout of the INA106 IC.

is a two-channel op-amp. The TL072 op-amp has the advantages of low power consumption, low offset currents, low noise, high slew rate which is $13 \mathrm{~V} / \mu \mathrm{s}$, and relatively low total harmonic distortion which is $0.003 \%$ [12] . These advantages make it a suitable candidate for amplifying the weak EMG signals.

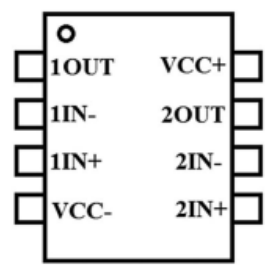

Fig. 2: Pin layout of the TL072 IC.

The microcontroller used for processing the EMG signal is Arduino Uno. The Arduino microcontroller is a free opensource platform widely used for developing and designing electronic based project. It is compatible with most of the available electronic devices in the market and is capable of transmitting and receiving data to and from the devices. According to [13], an Arduino can receive data from input devices such as sensors, antennas and potentiometers and transmit the data to output devices such as LEDs, speakers and motors. Therefore, Arduino Uno is used here as an interface to display the EMG signals at the computer. Figure 3 depicts the layout of the Arduino Uno board [14].

The Arduino Integrated Development Environment (IDE) is used to write and upload programs to Arduino Uno. As can be seen in Figure 4, the user interface of Arduino IDE is separated into three sections, namely, the command, coding, and message window areas. The command area comprises a list of function options. Here, the 'verify', 'upload', 'serial monitor', and 'serial plotter' functions are used to program the microcontroller. The 'verify' and 'upload' functions are used to compile and upload the code to the Arduino Uno board, while the 'serial monitor' and 'serial plotter' functions are used to display the output of the EMG signals. Programming is written at the coding area. Since a simplified version of the $\mathrm{C}++$ language is used for programming, users with basic knowledge on $\mathrm{C}$ or $\mathrm{C}++$ programming language should be able to pick up the skill with ease. There are two compulsory routines for every coding - namely the setup 'void setup ()' and loop 'void loop ()' routines. The setup routine is to initialize the program once the reset button on the Arduino Uno board is triggered. The loop routine, on the other hand, is used to notify the board to perform the task iteratively. The function of the message window area is to display the compilation process. Warning or error messages will be displayed in that area whenever the compiler comes across inaccurate syntaxes.

\section{B. EMG CIRCUIT}

The block diagram of the EMG circuit is shown in Figure 5. As shown in the figure, the EMG sig- 


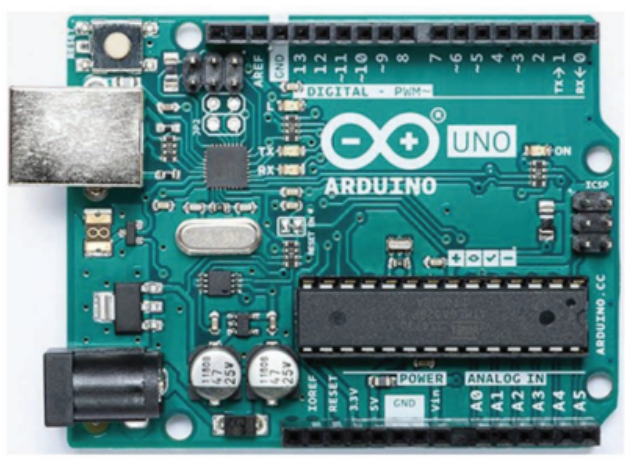

Fig. 3: The layout of the Arduino Uno board [14].

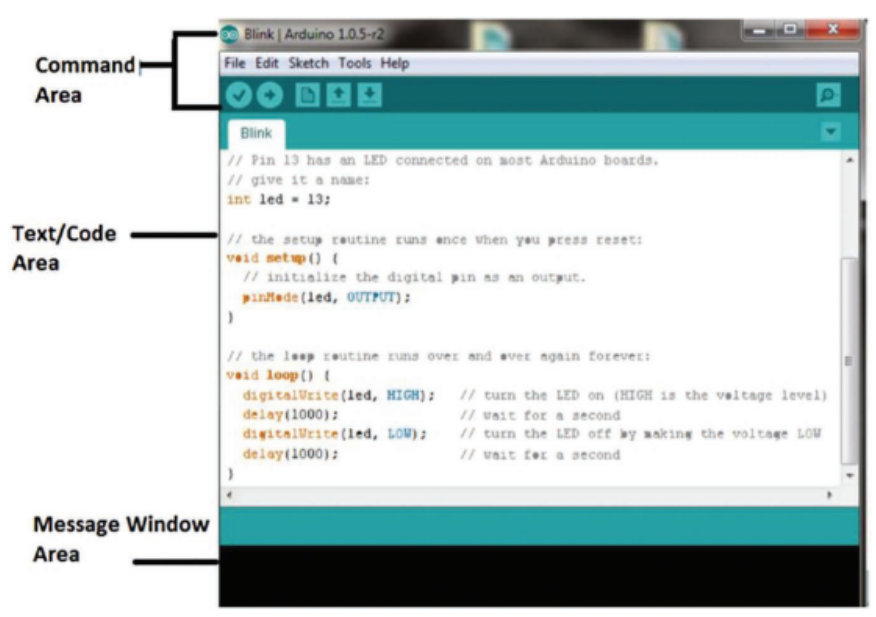

Fig. 4: The Arduino IDE.

nal has to undergo six stages of processes within the circuit. Firstly, the signal detected by the electrodes have to be compared and amplified by the differential op-amp. It has to subsequently go through two stages of amplification and signal faltering to optimize the SNR. The signal also has to be rectified, so that only positive DC voltage is delivered at the final output. Detailed elucidation of each stage is presented in this section.

As described at the early part of this section, EMG signals are highly susceptible to noise. Since charging and discharging a capacitor will contribute considerable electrical noise, power supplied from an IC regulator or a DC-to-DC converter is not suitable to be used as the source of the circuit. Instead, two 9 $\mathrm{V}$ dry cell batteries were used here. The schematic in Figure 6 illustrates the connection of the batteries to the EMG circuit.

Figure 7 depicts the circuit connection at the first stage of the EMG circuit. As can be seen from the figure, the electrodes are to be connected to the input pins of the INA106 differential amplifier. The signal at the second electrode is subtracted with that at the first electrode. This is to get rid of the common signal at both input signals, so that only clean signal is amplified at the output of the op-amp. The common signal is the ambient noise - the frequency of which resembles closely that of the nerve impulse [12]. The output amplifier gain A1 can be computed based on (2) below:

$$
A_{1}=\frac{R_{\text {sense }}+R_{1}}{R_{\text {input }}}
$$

where $R_{\text {sense }}$ and $R_{\text {input }}$ are, respectively, the 100 and $10 \mathrm{k} \Omega$ internal resistors in the INA106 IC, and $R_{1}$ is the external $1 \mathrm{M} \Omega$ resistor shown in Figure 7 . By substituting the values of the resistors into (7.2), gain $A_{1}$ is found to be 110 .

In the second stage, the signal goes through an inverting amplifier so as to produce a $180^{\circ}$ phase shifted amplified output. The configuration of the inverting amplifier is constructed using the TL072 op-amp. As can be observed in Figure 8, the inverting amplifier consists of a $150 \mathrm{k} \Omega R_{f}$ feedback resistor and a $10 \mathrm{k} \Omega R_{\text {in }}$ input resistor - both of which are connected to the inverting pin. The output signal at the first stage (i.e. the differential amplifier) is fed to the inverting pin. Gain $A_{2}$ of the inverting amplifier is 15 and can be found by substituting the values of the $R_{f}$ and $R_{\text {in }}$ into (7.3) below:

$$
A_{2}=-\frac{R_{f}}{R_{i}}
$$

The third stage of the EMG circuit constitutes a high pass filter. The output of the amplified signal in the second stage is connected to the capacitor in Figure 9. The function of the high-pass filter is to remove the noise created by motion artefact such as the movement of joints. As can be seen from the figure, two $150 \mathrm{k} \Omega$ resistors and a $0.01 \mu \mathrm{F}$ capacitor are used in the circuit. The purpose of introducing the capacitor at the input pin is to block DC signal from propagating into the circuit [12]. The cut off frequency $f_{c}$ of the filter can be calculated using (7.4)

$$
f_{c}=\frac{1}{2 \pi R C}
$$

where $R$ and $C$ are, respectively, the resistance and capacitance connected to the inverting input. Since noise created by motion artefact is typically below $10 \mathrm{~Hz}$, the cut off frequency $f_{c}$ is set to be at $106 \mathrm{~Hz}$. This is to say, only signals with frequencies higher than $106 \mathrm{~Hz}$ are allowed to propagate to the subsequent stage.

In the fourth stage, the filtered signal goes through full wave rectification. As shown in Figure 10, the components involved in building the rectifier include four $10 \mathrm{k} \Omega$ resistors, two pn-junction diodes, and two op-amps. The rectifier converts signals in the negative phase to the positive phase, i.e., the $\mathrm{AC}$ input signal is changed to DC signal 


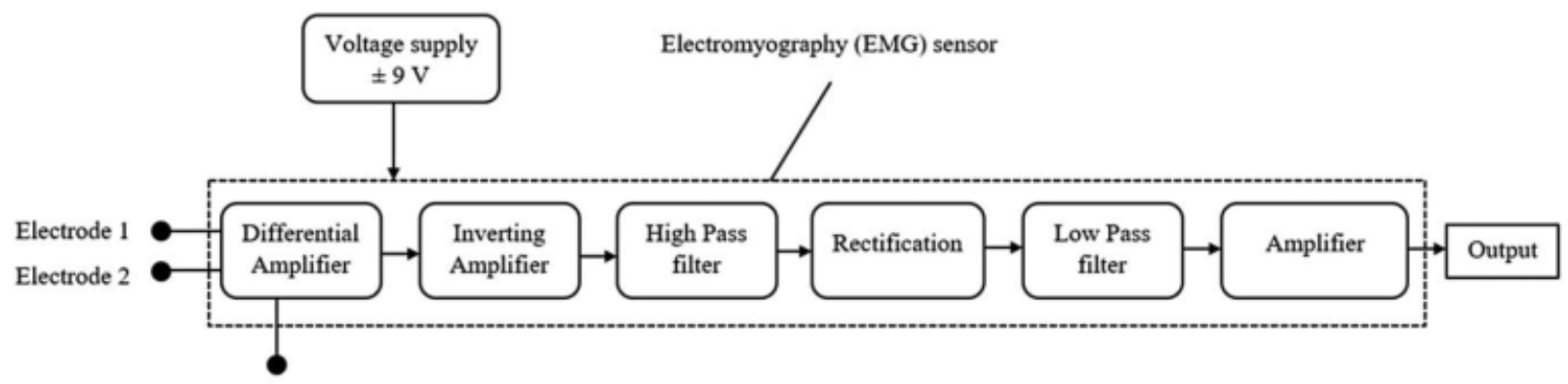

Reference electrode

Fig. 5: Block diagram of the EMG sensor.

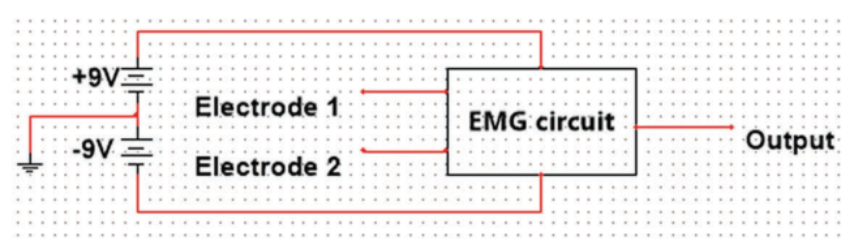

(a)

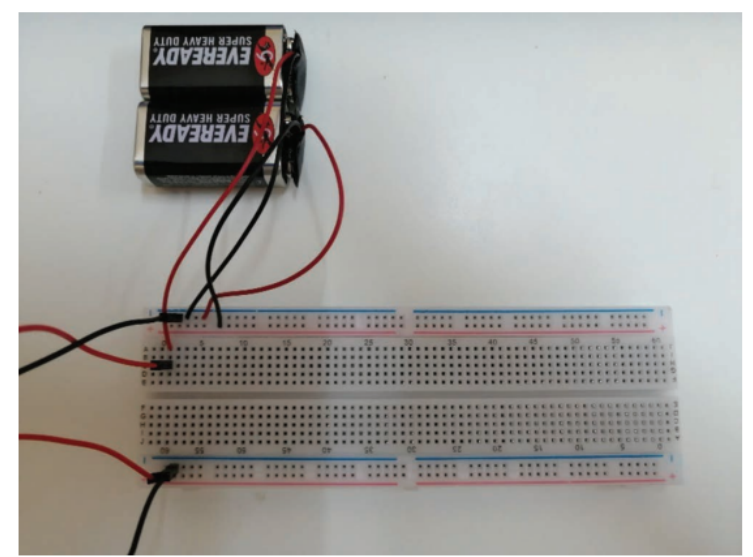

(b)

Fig. 6: (a) The voltage supply circuit and (b) the connection of the voltage supply to a breadboard.

at the output. In the fifth process step, the rectified signal is fed to a low pass filter. The function of the low-pass filter is to remove the effect of aliasing high frequency components [12]. The filter at this stage is developed by connecting two passive components - a $1 \mu \mathrm{F}$ capacitor and an $80.6 \mathrm{k} \Omega$ resistor - at the feedback loop and another $80.6 \mathrm{k} \Omega$ resistor to the inverting input. The schematic of the low pass filter is illustrated in Figure 11.

At the final stage, the EMG signal is amplified to a desirable detectable value. Most often than not, the EMG signal generated by the contraction of muscles is extremely weak. Hence, an amplifier with high gain is necessary to increase the output voltage to a reasonable magnitude. Here, the TL072 op-amp is again employed for this purpose.

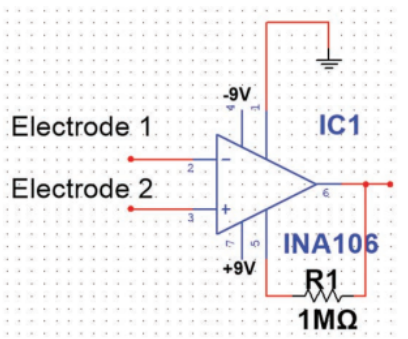

(a)

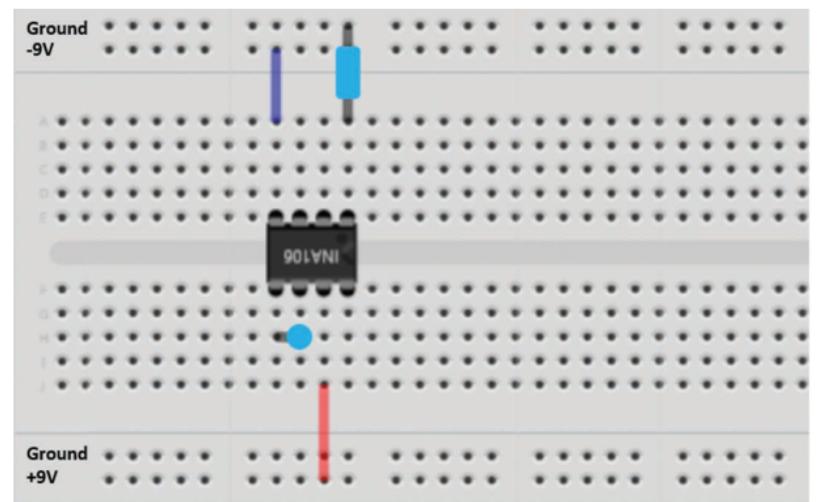

(b)

Fig. 7: (a) The circuit schematic of the INA106 differential amplifier and (b) its connection on a virtual breadboard.

As can be seen in Figure 12, a $1 \mathrm{k} \Omega$ resistor and a $100 \mathrm{k} \Omega$ trimmer potentiometer are connected to the inverting input. The trimmer potentiometer is used to vary gain $A_{3}$ of the amplifier [12]. The gain is in direct proportion with the resistance of the potentiometer. Since the circuit configuration of this amplifier is similar to that in the second stage, gain $A_{3}$ can be computed by dividing the value of the feedback resistor with the $1 \mathrm{k} \Omega$ input resistor.

In a nutshell, the complete EMG circuit is constructed by combining the circuit networks from the first stage to the final stage, i.e., from Figures $7.6-7.12$. Figure 13 illustrates the schematic of 


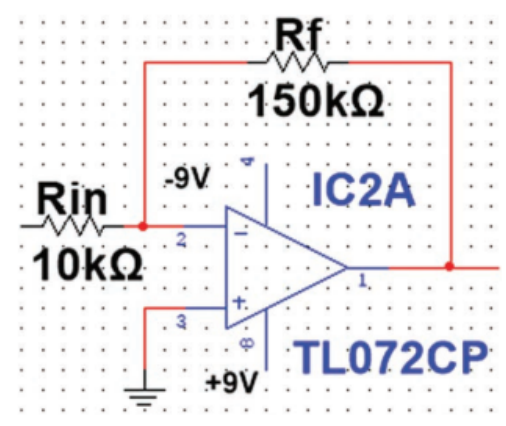

Fig. 8: The circuit schematic of the inverting amplifier.

the overall EMG circuit and its components' connections on a virtual breadboard.

\section{SIGNAL AND DATA ACQUISITION}

In order to analyze the efficacy of acupuncture, the data measured before and after the treatment is to be stored and analyzed. The Arduino Uno microcontroller acts like a circuit interface between the EMG sensor and the display (which is a computer in this case). The microcontroller records the EMG signal in digital form and display them onto the screen. Since Arduino Uno can only support analog input signal ranging from 0 to $5 \mathrm{~V}$, care must be taken to ensure that the output signal amplified at the final stage of the EMG circuit is below $5 \mathrm{~V}$. To perform data acquisition, the output signal from the EMG sensor is to be connected to the analog input pin of the Arduino Uno board and the ground wires of both devices are to be connected together, as shown in Figure 14.

The output of the Arduino Uno board is to be connected to the USB port of a computer. Once the hardware has been set up, the Arduino IDE is launched, and the code for displaying the result of the EMG signal is compiled and uploaded to the Arduino Uno board. A snapshot of the code used in this project is depicted in Figure 15(the complete Arduino code is listed in the Appendix). In the Arduino code, the analog input pin A 0 is used to receive the output generated by the EMG sensor.

Analog-to-digital conversion (ADC) is then performed so that the output value can be expressed in digital form $V_{d}$. Arduino Uno is equipped with a 10 -bit analog to digital converter. This means that a 0 and $5 \mathrm{~V}$ operating voltage return the value of 0 and 1023, respectively. In order to express the digital value $V_{d}$ in terms of voltage $V_{s}, V_{d}$ which accounts a fraction of the total 1023 is multiplied by $5 \mathrm{~V}$, i.e.

$$
V_{s}=\frac{V_{d}}{1023} \times 5 \mathrm{~V}
$$

The voltage $\mathrm{Vs}$ is then compared with a threshold value, which is set at a value close to half of

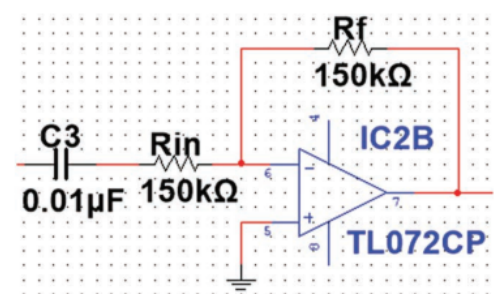

(a)

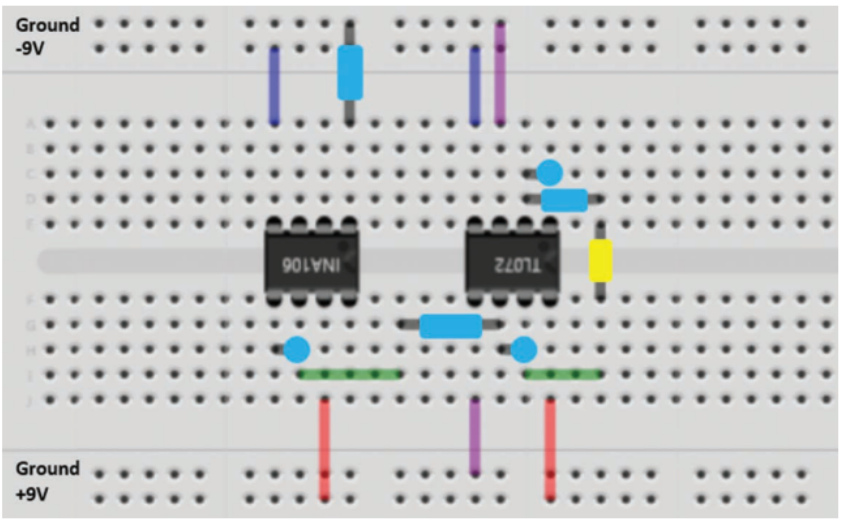

(b)

Fig. 9: (a) The circuit schematic of a high-pass filter and (b) its connection to the differential and inverting amplifers on a virtual breadboard.

its amplitude, i.e., $2 \mathrm{~V}$. If the voltage value exceeds the threshold value, the on-board LED will be switched on, and the 'CONTRACT!' message will appear on the serial monitor tool of the Arduino IDE. On the contrary, if value $\mathrm{Vs}$ is equal or lower than the threshold value, the on-board LED will be switched off and the 'RELAX!' message will be displayed. Once the code is uploaded to the board, the complete system is in immaculate readiness.

Before starting the measurement process, it is essential to ensure that the surface electrodes are appropriately placed. Once the target muscle has been identified, one surface electrode is to be adhered to the middle part of the muscle, while another electrode is to be adhered to the end of it. A third surface electrode which serves as the reference is to be adhered to the bony part which is in close proximity to the target muscle. It is to be noted that, the third reference electrode must be avoided from adhering to any parts with muscles since this will result in inaccuracy in the data acquiring process.

An example of how the electrodes are adhered to measure the EMG signal from the bicep muscle is shown in Figure 16. Figures 7.17 and 7.18 illustrate the display on the serial monitor and serial plotter when the bicep muscle is fixed and released. As can be seen from the figures, the serial monitor is capable of displaying the status of the muscle (i.e. either contracting or relaxing) and the voltage 


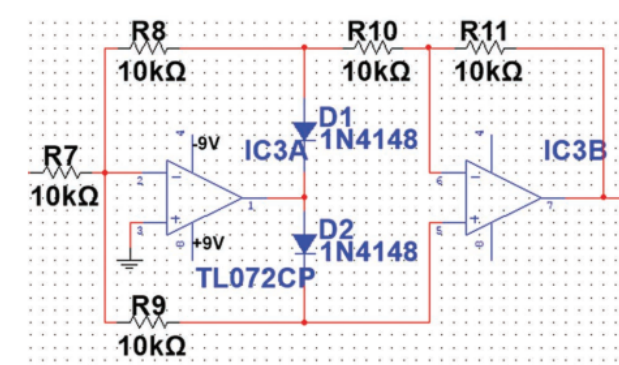

(a)

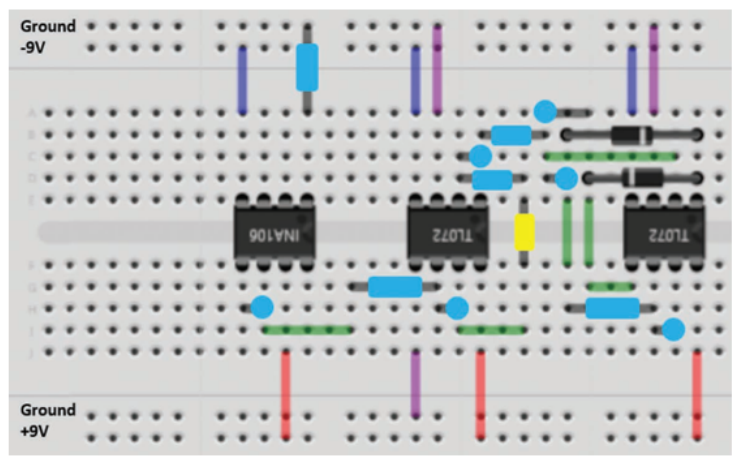

(b)

Fig. 10: (a) The circuit schematic of a full wave rectifer and (b) its connection to the high pass filter and amplifiers on a virtual breadboard.

value of the EMG signal. Apart from the display on the serial monitor and serial plotter, the status of the muscle can also be observed from the Arduino Uno board. As shown respectively in Figures 7.19 and 7.20 , the on-board LED will be switched on when the muscle is in contract condition and, it will be switched off otherwise.

\section{TEST PROCEDURES}

Four volunteers had been involved in the acupuncture case study, with each of them suffering from muscle impairment at different parts of the bodies. The first volunteer suffered from neck pain, the second suffered from calf muscle cramp due to excessive exercises and the third and fourth suffered from thigh muscle pain. The patients were treated by an acupuncturist who has had more than 10 years of clinical experience. The patients were rested on a treatment couch before the acupuncturist started carefully locating the areas of the injured muscles by palpation. According to Tsuei [1], each meridian is related to a specific organ. Hence, once the injured areas were identified, the meridians that went through those areas were traced. Acupuncture needles were subsequently inserted into the distal acupoints. Very often, when a needle was pricked, the acupuncturist would consult the patients if the needling sensation could be felt. According to the acupuncturist, once the needle reaches its intended depth and depending on the meridians be-

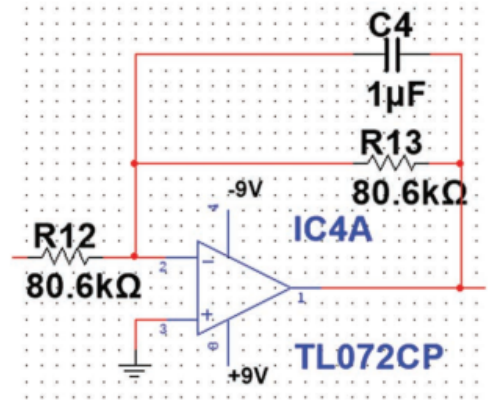

Fig. 11: The circuit schematic of the low pass amplifier.

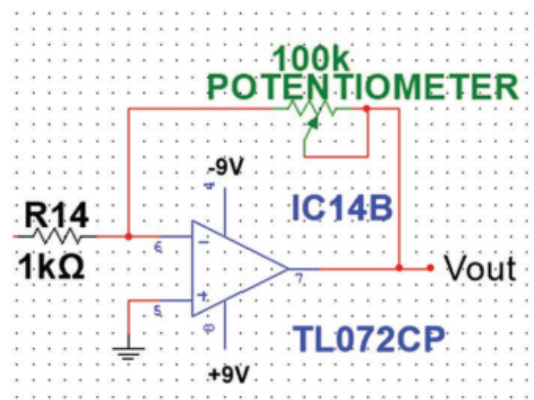

Fig. 12: The circuit schematic of the inverting amplifer connected to the last stage of the EMG circuit.

ing triggered, the patient should feel either a mild tingling sensation or a glitch of electric sensation. If the sensation is not felt immediately, it is elicited via needle rotation.

Since changes in the muscle length and movement may easily induce motion artefact, the EMG activity was measured during a 10-second, sub-maximal, isometric contraction. The reading at each second before and after the treatment was recorded. In order to minimize measurement errors, the EMG activity at each second was measured twice and the average was taken. Once the entire therapeutic process had fnished, the EMG signals of the first three patients were measured immediately, while the fourth were measured 30 minutes afterward. Again, the EMG signal after the therapy was measured at each second for a consecutive duration of 10 seconds. Figures 21 and 24 illustrate the acupuncture therapies administered on the four patients, whereas Figures 7.25 shows the EMG measurement process performed after the therapy. Although the EMG activity was observed throughout the process (i.e. before, during, and after the therapy), only the results before and after the treatment were saved, compiled, and analyzed (Figures 22 and 23.). 


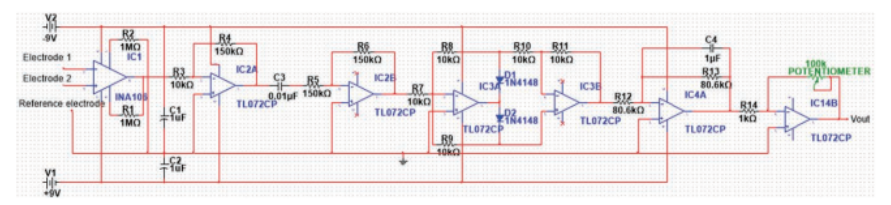

(a)

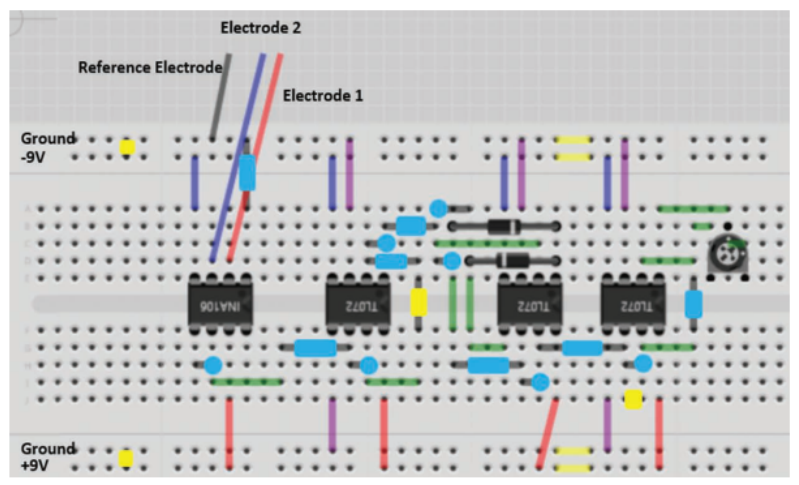

(b)

Fig. 13: (a) The complete circuit schematic of the EMG sensor and (b) the connections of the components on a virtual breadboard.

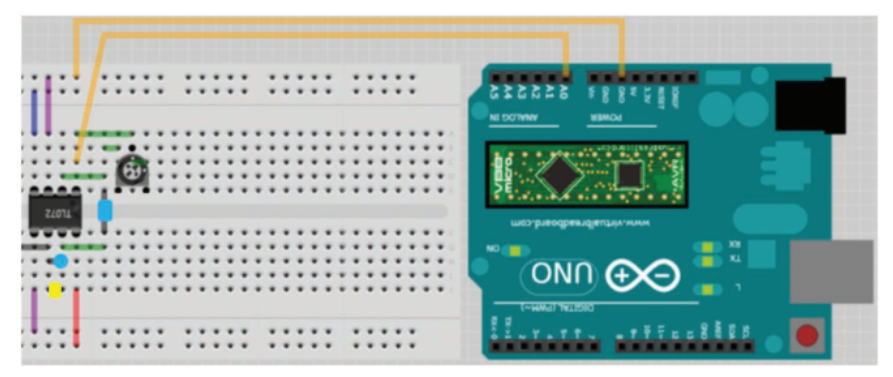

Fig. 16: Placement of the surface electrodes on the

Fig. 14: Connection of the EMG circuit to Arduino Uno on a virtual breadboard.

\section{RESULTS AND DISCUSSION}

Figures 26-29 and 30-33 show, respectively, the EMG signals of the four volunteers before and after the acupuncture treatments. Clearly, the signals are constantly fluctuating since they are highly sensitive to electrical noise - in particular, motion artefact and the inherent instability of the signal. Hence, a variation within $\pm 15 \%$ from its average value would be regarded as a normalcy and would not be considered as the after effect of acupuncture.

The average EMG signal before the acupuncture treatment and the signal at each second after the treatment for a duration of 10 seconds are depicted in Figures 7.34-7.37. It is to be emphasized again, here, that the measurements for the first three volunteers were taken instantly after the treatment; while, that for the last volunteer were taken half an hour later. Upon close inspection on the figures, bicep muscle.

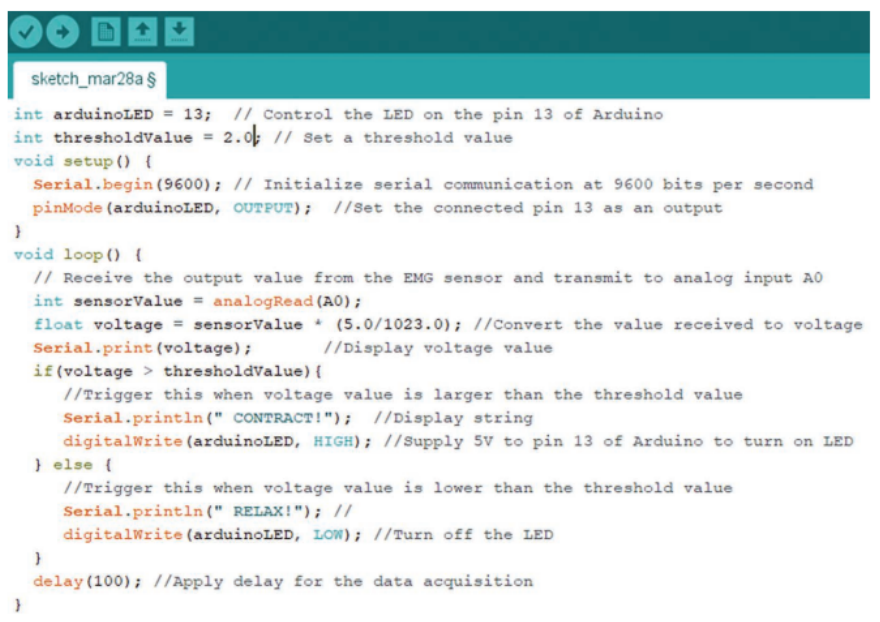

Fig. 15: Arduino code for data acquisition.

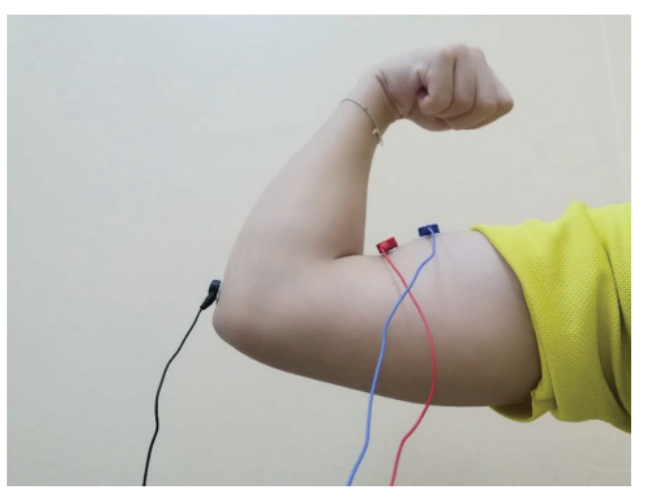

it can be observed that the EMG signal of the four volunteers before the acupuncture treatment are all above the $2 \mathrm{~V}$ threshold, with the third volunteer having the highest magnitude (i.e. $3.58 \mathrm{~V}$ ) and the second having the lowest magnitude (i.e. $2.56 \mathrm{~V}$ ). This is to say that their muscles were suffering from contractions due to injuries, and it is very likely that the third volunteer had the most severe injury, while the second the least severe. It can also be seen that the posttreatment EMG signals for the first three volunteers resemble closely those before the treatments - their average magnitudes after the treatments only vary, respectively, by $5.97 \%, 9.06 \%$, and $4.55 \%$ compared to the pretreatments. Although the posttreatment signals for the second and third volunteers evince slight reductions, the discrepancies are still within the $15 \%$ range. Hence, the differences are very likely a result of electrical noise coupling effects.

On the other hand, the average post treatment magnitude for the fourth volunteer drops by 


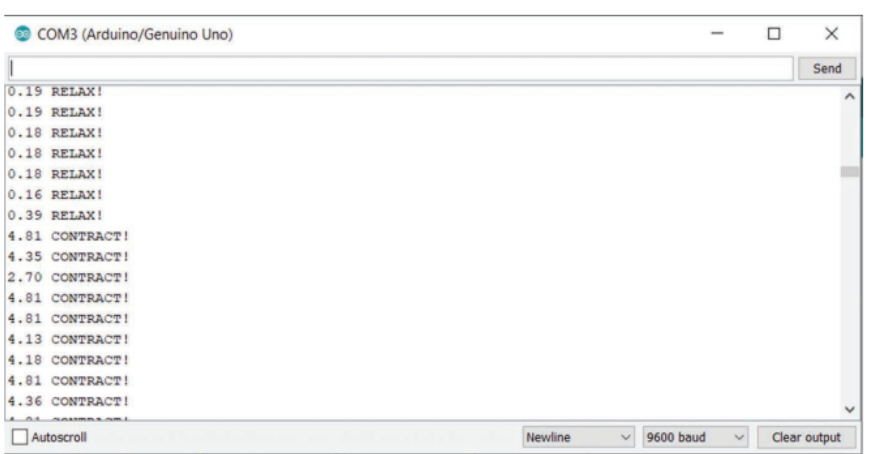

Fig. 17: The voltage values generated during muscle contraction and the status of the muscles are displayed on the serial monitor of the Arduino IDE.

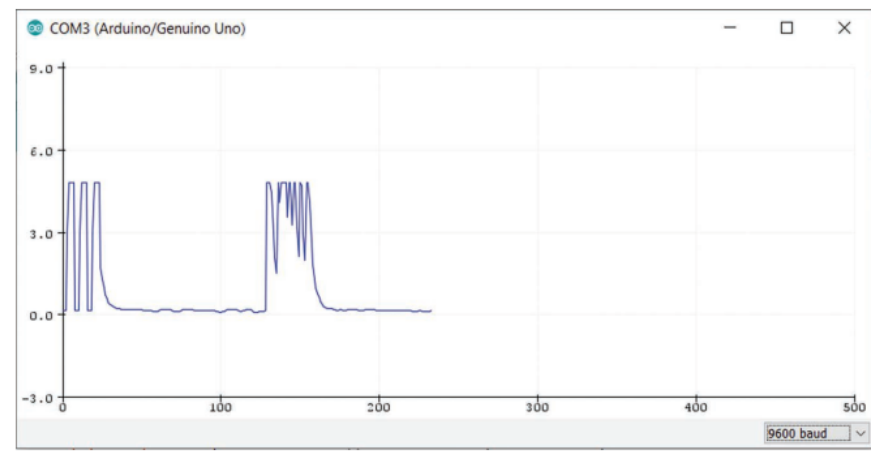

Fig. 18: EMG signal generated by muscle contraction is displayed on the serial plotter of the Arduino IDE.

$26.25 \%$. The reduction rate is conspicuous and could be easily observed from paper Figure 37 . Despite remaining above the threshold, it is apparent that the muscles of the fourth volunteer have manifested signs of relaxation after the treatment. The observation suggests strongly that acupuncture does provide effective medication to the patients. The effect of the treatment, however, may not be immediate and may, in fact, only slowly but progressively become noticeable.

\section{HYPOTHESIS}

According to $[15-18]$, more than $90 \%$ of the skin points which are of particularly high conductivity coincide with the acupoints. A general conjecture for this phenomenon is that the water clusters in the meridians conduct better than proteins [2]. Solutions which contain these water clusters were found to have an electromotive force (emf) in the range of $10-100 \mathrm{mV}[19-23]$ and, in fact, the emf at the acupoints is higher by the order of $10 \mathrm{mV}$ in comparison with the neighboring skin [2]. Hence,

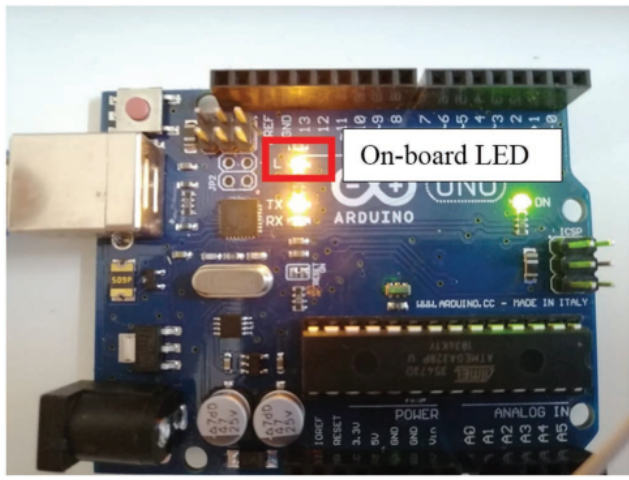

Fig. 19: The on-board LED will be switched on when the muscle contracts.

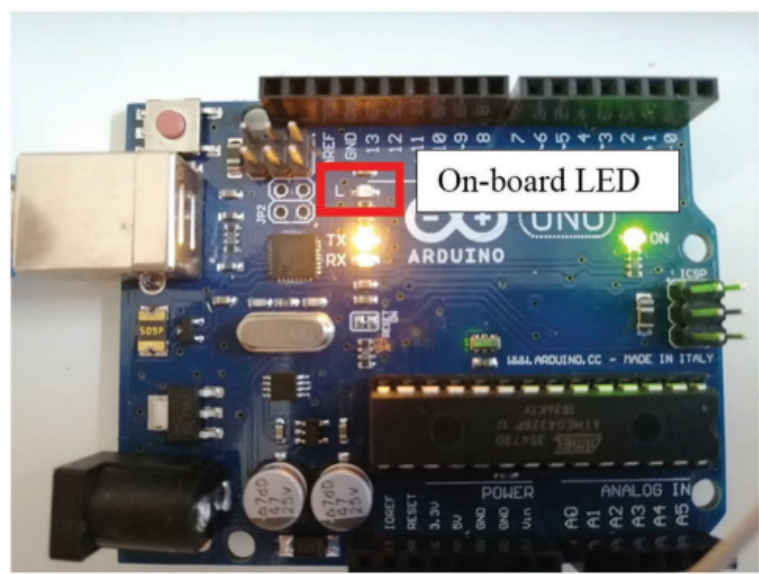

Fig. 20: The on-board LED will be switched off when the muscle relaxes.

electromagnetic waves travel faster through meridians than through nonmeridian tissue [15]. The assert given by [15] is corroborated by the findings in [24-26] which demonstrated that meridians are good paths for electric current.

Now, if one hypothesizes the entire meridian system to operate like an electric circuit network, then one can think of the injured parts as those related to the meridians where the conductivities have dropped. This is to say that an anomaly has occurred to the water clusters in those meridians, resulting in an unusual increase in resistance. The high resistance hampers the propagation of the polarized $q i$. When the improper distribution of the electrons (due to the unregulated $q i$ ) exceeds the intrinsic tolerance of a human body, one starts to feel uncomfortable or ill [27]. To allow sufficient amount of $q i$ to flow through, it is apparent that alternate paths with much lower resistance are required. Most acupuncture needles are made of stainless steel; while some are made of silver - both of which are highly conducting materials. Inserting the acupuncture needles to the acupoints can 


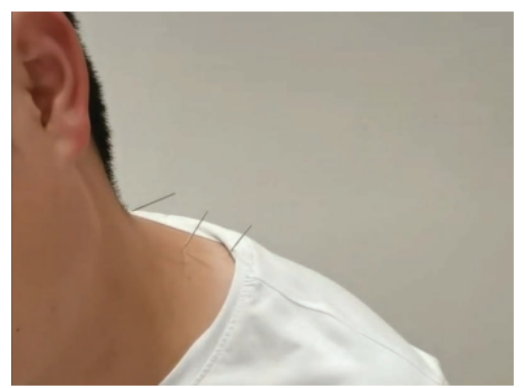

Fig. 21: Acupuncture is administered on the first volunteer to remedy his neck pain.

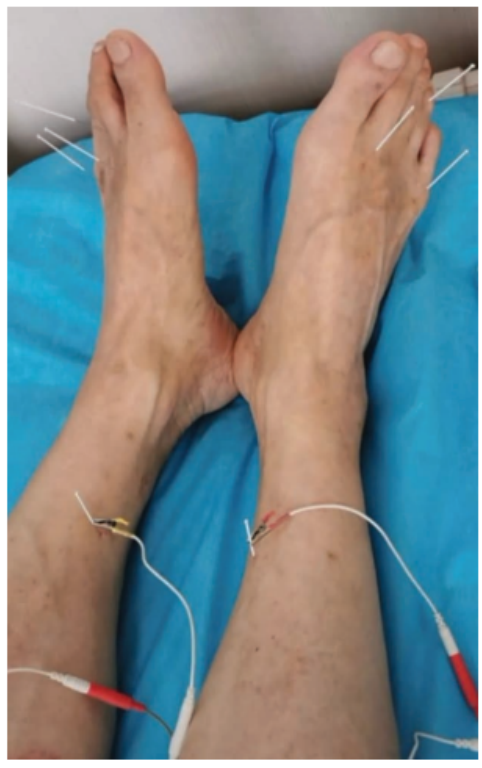

Fig. 22: Acupuncture is administered on the second volunteer to remedy his calf muscle cramp.

be conceived as connecting parallel wires between the nodes with high resistance. A short-circuited path is therefore created at the abnormal meridians. Physically, this actually means that the needles are changing the constitutive properties of the body $[28,29]$ within the region of the abnormal meridians. In other words, not only the conductivity but the permeability and permittivity which dictates, respectively, the magnetic and electric field densities at the meridians will be altered as well. It is to be noted, however, that this change is physiological and therefore the pace may be rather gradual. Once the constitutive properties of the meridians start to return to their healthy values, albeit not yet fully restored, the flow of $q i$ would then be unclogged, and the imbalances in $q i$ are regulated. The hypothesis is in congruence with the results observed in the case study conducted in this work. It is therefore safe to assert that acupuncture indeed provides curative efficacy, although the effect is not

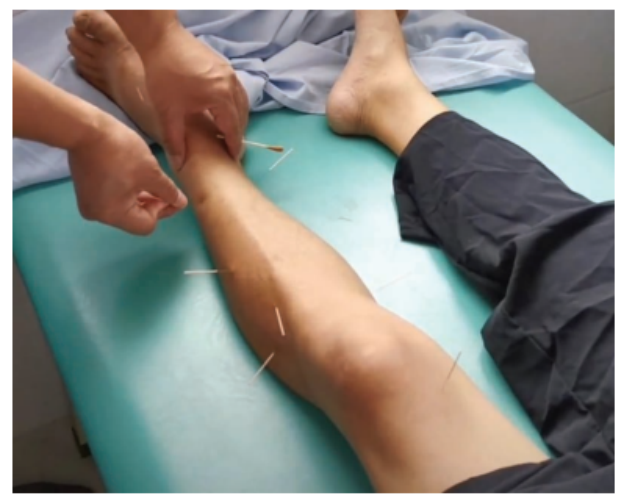

Fig. 23: Acupuncture is administered on the third volunteer to remedy his thigh muscle impairment.

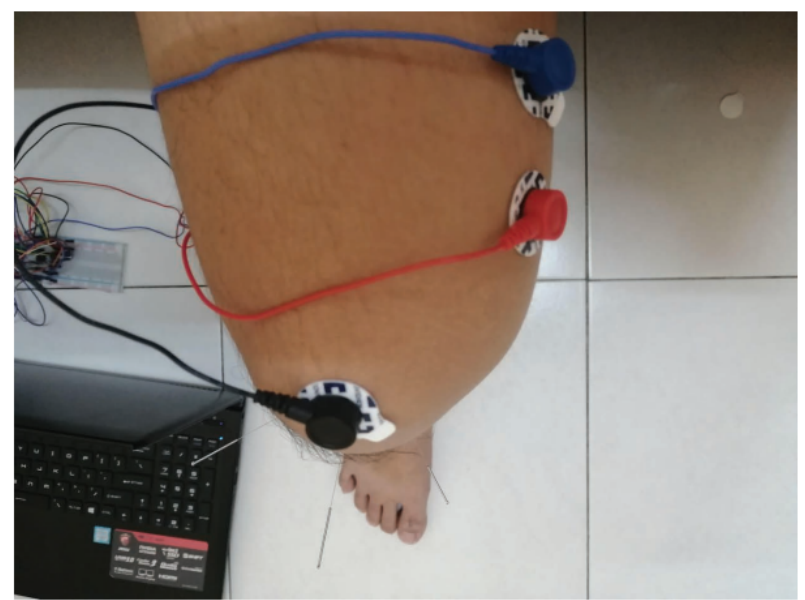

Fig. 24: Acupuncture is administered on the fourth volunteer to remedy his thigh muscle impairment.

immediate.

\section{CONCLUSiON}

In this paper, the efficacy of acupuncture therapy has been investigated. An EMG sensor was built to assess the effect of the acupuncture treatment. The sensor consists of electrodes, amplifiers, filters, and a rectifier. To detect the muscle activity, the electrodes of the sensor were adhered to the skin of the volunteers. The output signal of the sensor subsequently went through an ADC process and was finally displayed at the serial monitor and the serial plot. The measurement results indicate that by helping to regulate the imbalances in qi, acupuncture produces remedy effect to the patients. The effect is, however, not immediately evident. Hence, the patients may only feel the remedy effect slowly but progressively.

\section{APPENDIX}

int arduinoLED $=13$; 


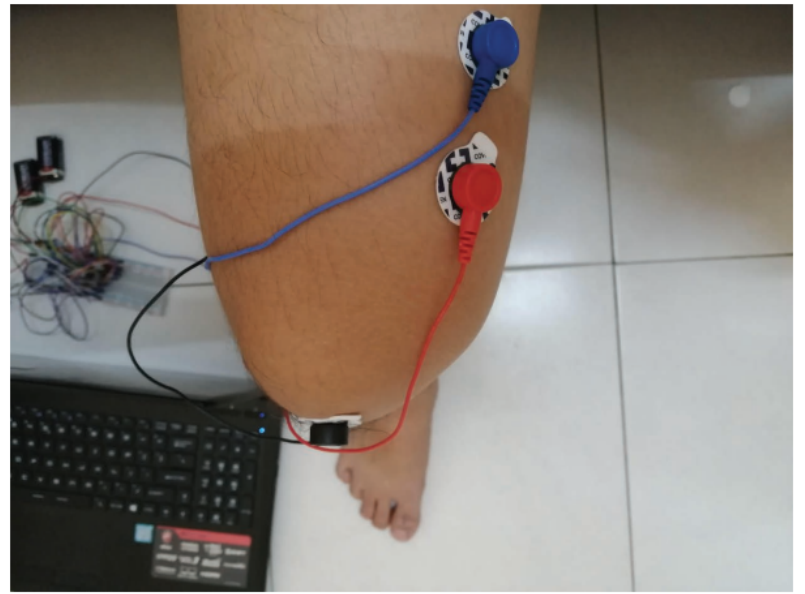

Fig. 25: The EMG readings are acquired after the acupuncture treatment.

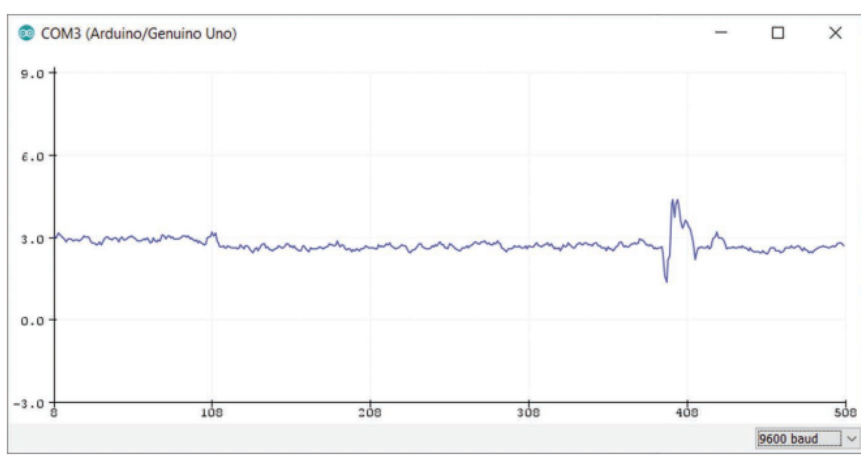

Fig. 26: The EMG signal of the frst volunteer before the acupuncture treatment.

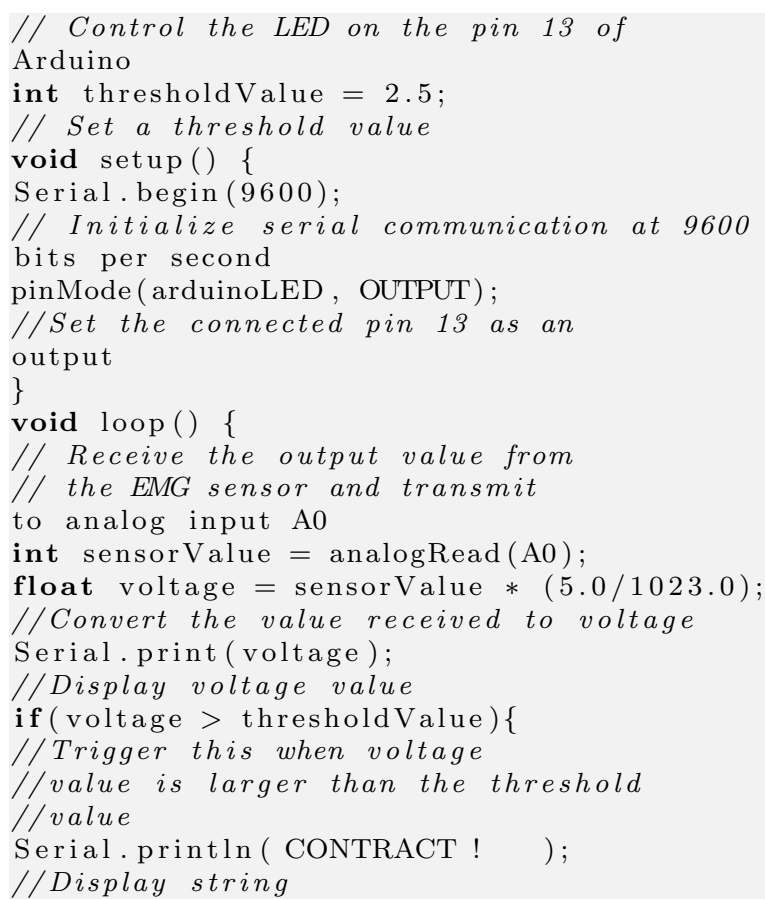

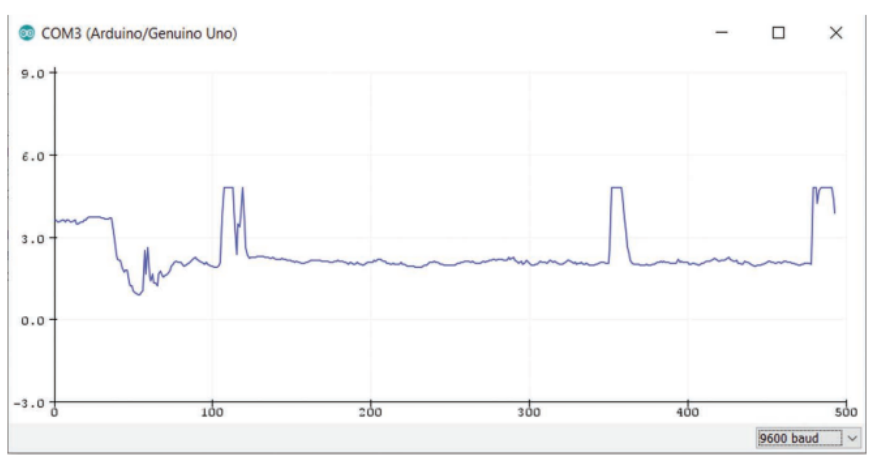

Fig. 27: The EMG signal of the second volunteer before the acupuncture treatment.

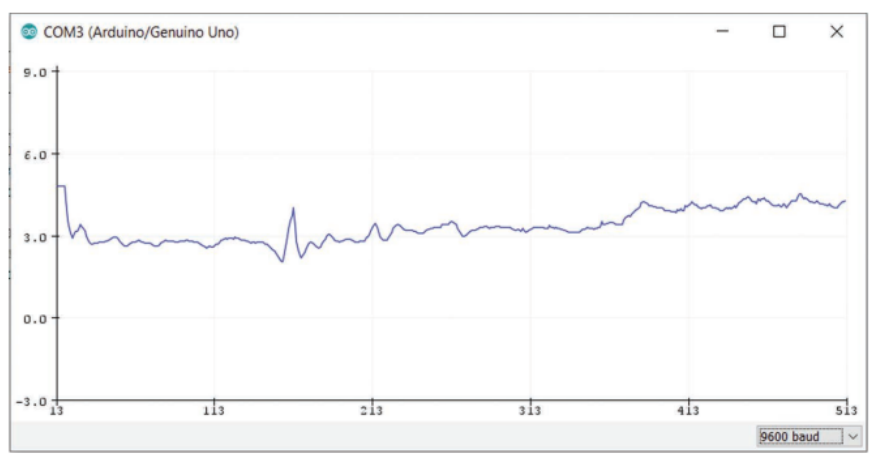

Fig. 28: The EMG signal of the third volunteer before the acupuncture treatment.

digitalWrite (onboardLED, HIGH);

//Turn on LED

\} else \{

/ Trigger this when voltage

//value is lower than the

/ threshold value

Serial.println( RELAX ! ); //Dislpay string digitalWrite (onboardLED, LOW); //Turn off LED \} delay $(100)$

//Apply delay for

//the data acquisition

\}

\section{ACKNOWLEDGMENT}

This work was supported in part by the Universiti Tunku Abdul Rahman research fund (project: IPSR/RMC/UTARRF/2020-C1/Y03). 


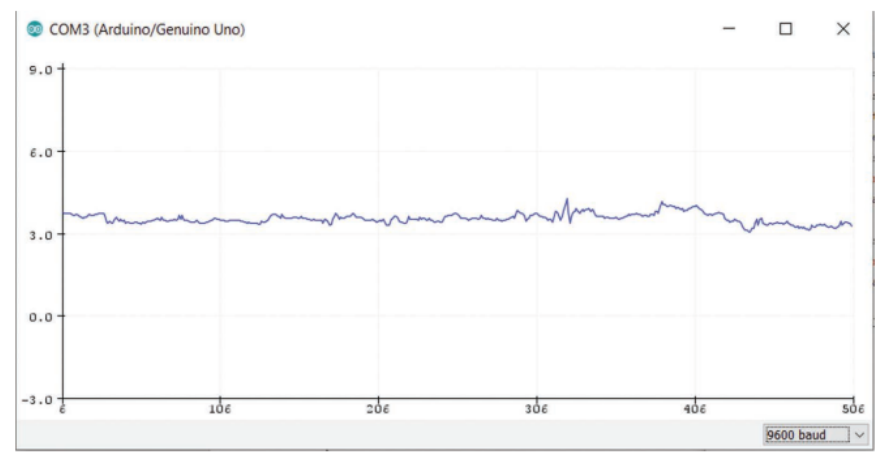

Fig. 29: The EMG signal of the fourth volunteer before the acupuncture treatment.

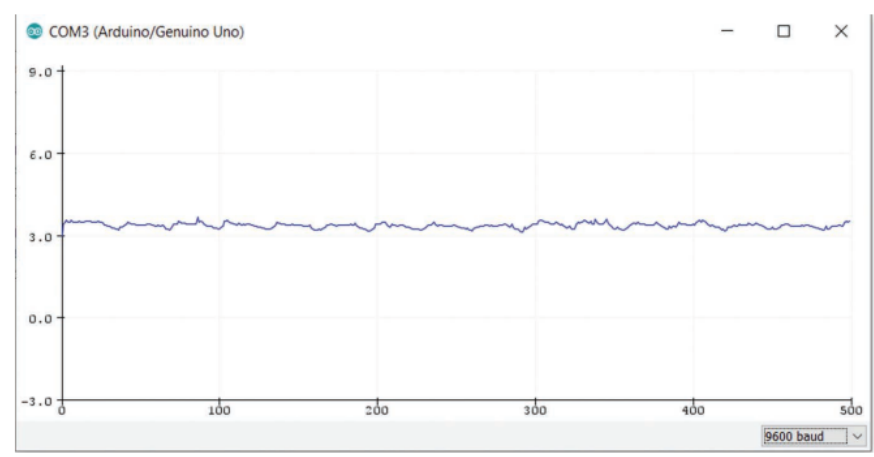

Fig. 30: The EMG signal of the first volunteer after the acupuncture treatment.

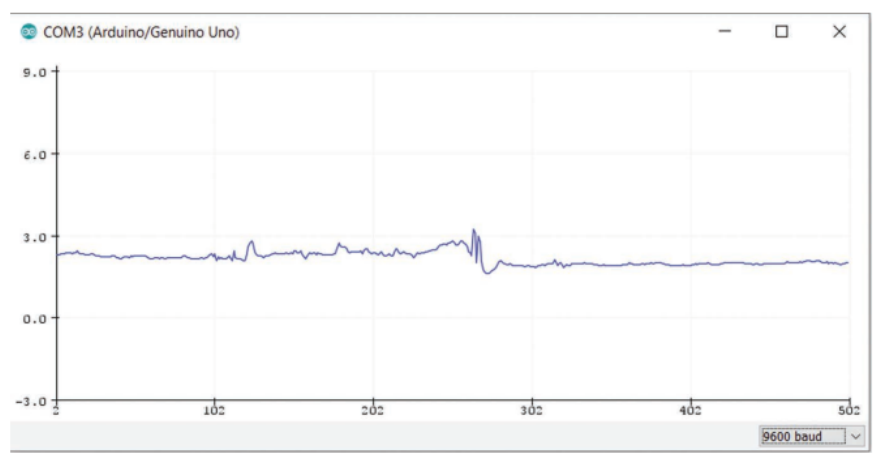

Fig. 31: The EMG signal of the second volunteer after the acupuncture treatment.

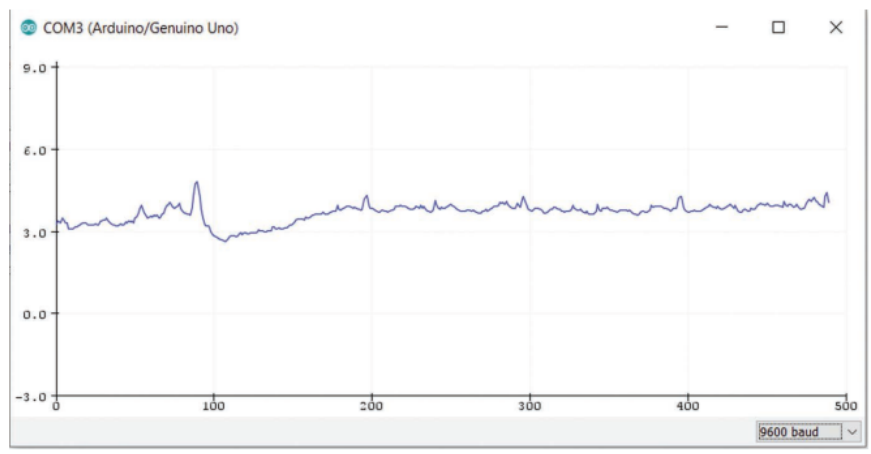

Fig. 32: The EMG signal of the third volunteer after the acupuncture treatment.

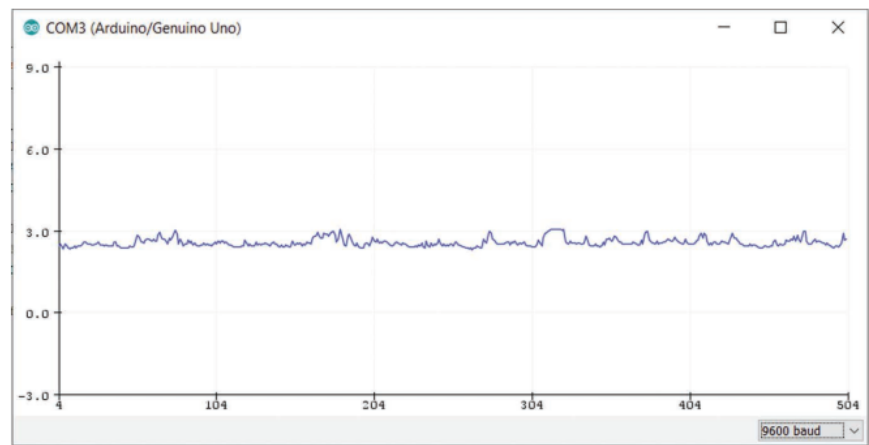

Fig. 33: The EMG signal of the fourth volunteer after the acupuncture treatment.

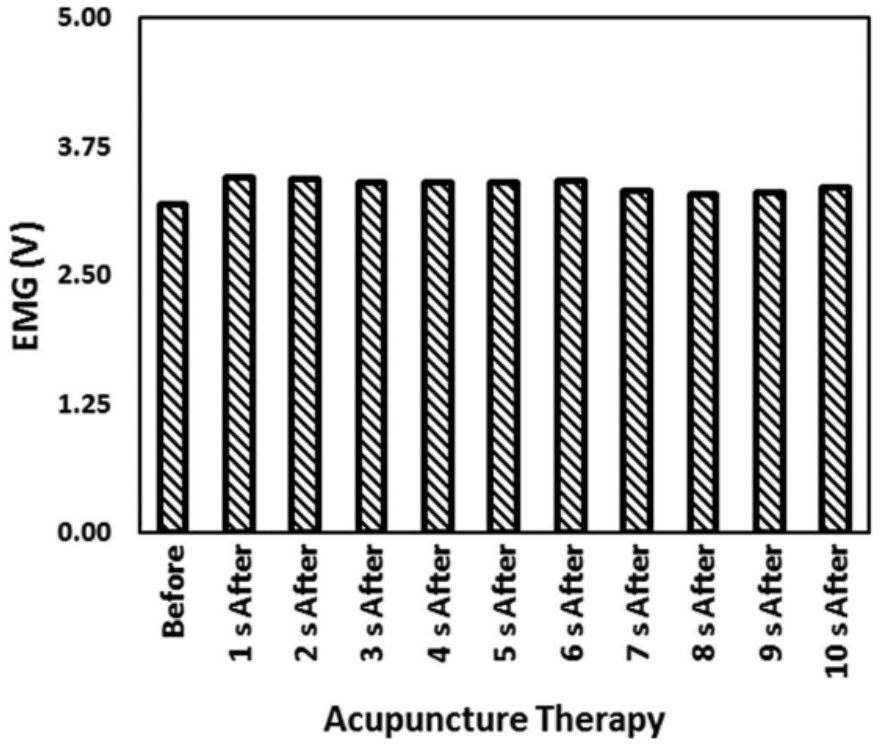

Fig. 34: Comparison of the EMG signal of the first volunteer before and after the acupuncture treatment. 


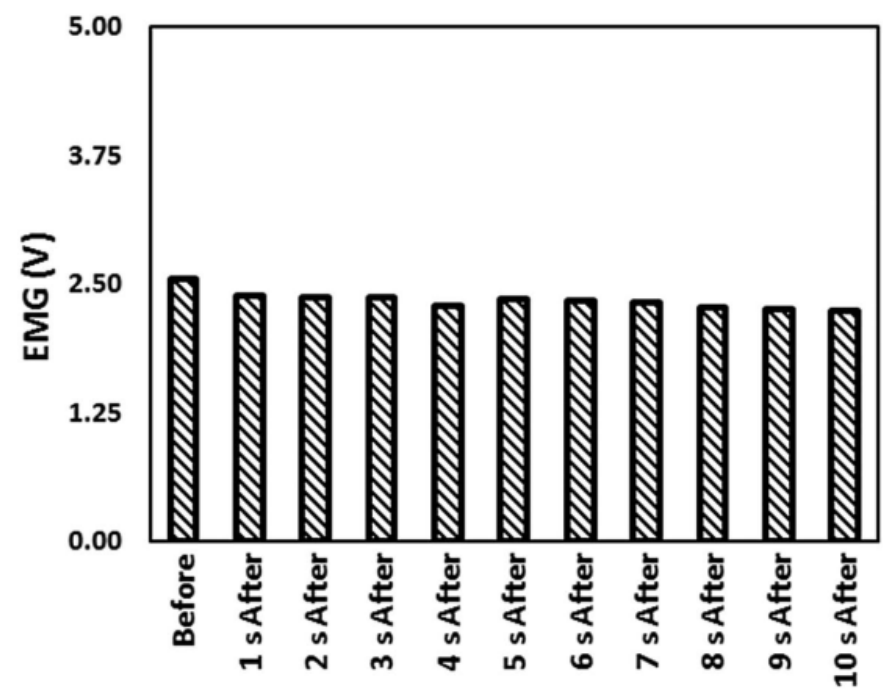

Acupuncture Therapy

Fig. 35: Comparison of the EMG signal of the second volunteer before and after the acupuncture treatment.

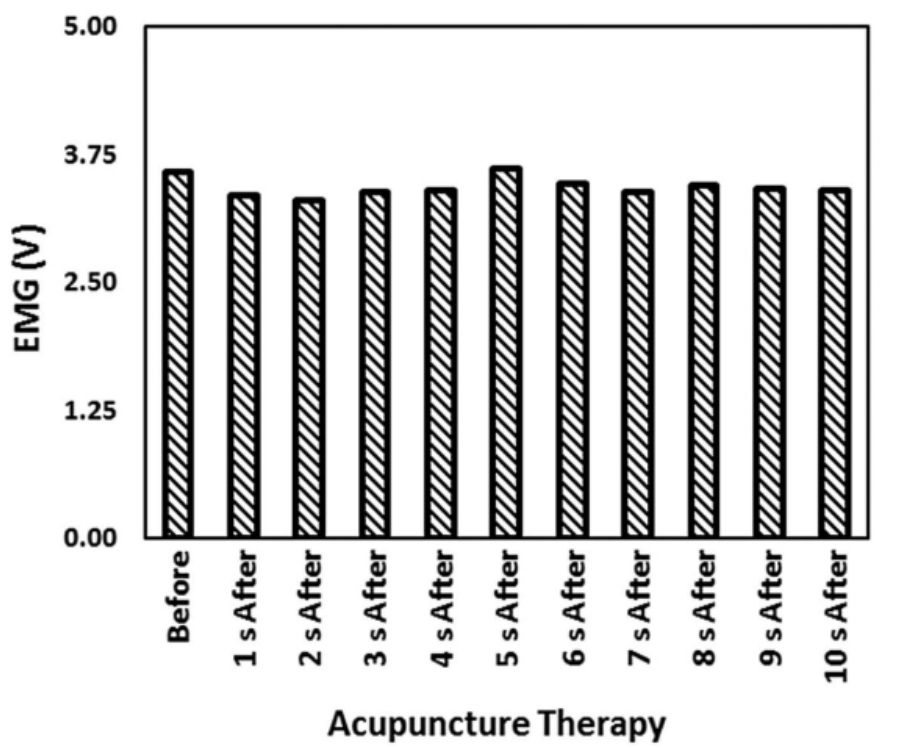

Fig. 36: Comparison of the EMG signal of the third volunteer before and after the acupuncture treatment.

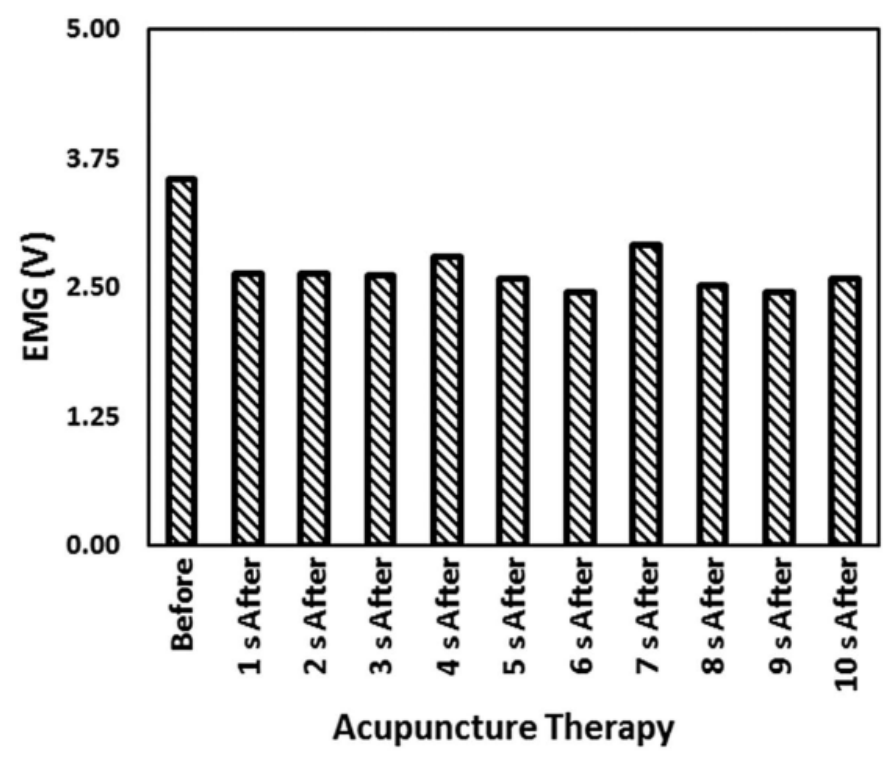

Fig. 37: Comparison of the EMG signal of the fourth volunteer before and after the acupuncture treatment.

\section{REFERENCES}

[1] Tsuei, J. J. 1996 The science of acupuncture-theory and practice, IEEE Eng Med Biol Mag 15, 52\{57.

[2] Lo, S.-Y. 2002 Meridians in acupuncture and infrared imaging, Med Hypotheses 58, 72\{76.

[3] de Camargo P. S., Lima C. R., Rezende M. L. A. E., Santos A. T. S., Hernandez J. W. R., and Silva A. M. 2018 The e_ect of auricular and systemic acupunc- ture on the electromyographic activity of the trapez- ius muscle with trigger points $\mid$ A pilot study, J Acupunct Meridian Stud 11, $18\{24$.

[4] Yeung A., Chan J. S. M., Cheung J. C., and Zou L. 2018 Qigong and Tai-Chi for mood regulation, Focus 16, $40\{47$.

[5] Tsuei J. J., Lam F. M. K., and Chou P. 1996 Clinical applications of the EDST, IEEE Eng Med Biol Mag 15, $67\{75$.

[6] Tough L. 2006 Lack of e_ect of acupuncture on electromyo-graphic (EMG) activity \{ A randomised controlled trial in healthy volunteers, Acupunct Med 24, $55\{60$.

[7] Farida I. M., Ismail G., Susanti H., and Prasetio M. E. 2011 Observation of acupuncture stimulation on human stamina improvement using EMG. 2nd IEEE International Conference on Instrumentation Control and Automation, pp. 71 \{76, Bandung, Indonesia.

[8] Reaz M. B. I., Hussain M. S., and Mohd-Yasin F. 2006 Techniques of EMG signal analysis: Detec- tion, processing, classifcation and applications, Biol Proced Online 8, $11\{35$. 
[9] Webster J. G. 1990 Encyclopedia of Medical Devices and Instrumentation. John Wiley Sons, Inc., Hobo- ken, NJ

[10] Advancer Technologies. 2011. DIY Muscle Sensor / EMG Circuit for a Microcontroller. Instructables workshop. https://www.instructables.com/MuscleEMG-Sensor-for-aMicrocontroller/ (accessed July 12, 2020).

[11] Jamal M. Z. 2012 Signal acquisition using surface EMG and circuit design considerations for robotic prosthesis, Comput Intell Electromyogr Anal. Per- spect. Curr. Appl. Future Challenges 18, $427\{448$.

[12] Desa H. M., Zuber M. S., Jailani R., and Tahir N. M. 2016 Development of EMG circuit for detection of leg movement. IEEE Symposium on Computer Applications Industrial Electronics, pp. 46\{51, Penang, Malaysia.

[13] Margolis M., Jepson B., and Weldin N. R. 2020 Arduino Cookbook: Recipes to Begin, Expand, and Enhance Your Projects. O'Reilly Media, Newton, MA.

[14] https://store.arduino.cc/usa/arduino-uno-rev3.

[15] Chen K.-G. 1996 Electrical properties of meridians, IEEE Eng Med Biol Mag 15, 58 \{63.

[16] Zhu Z. 1981 Research advances in the electrical specifcity of meridians and acupuncture points, Am J Acupunct 9, 203.

[17] Reichmanis M., Marino A. A., and Becker R. O. 1975 Electrical correlates of acupuncture points, IEEE Trans Biomed Eng 6, $533\{535$.

[18] Reichmanis M., Marino A. A., and Becker R. O. 1976 DC skin conductance variation at acupuncture loci, Am J Chin Med 4, $69\{72$.

[19] Holtzclaw B. J. 1998 New trends in thermometry for the patient in the ICU, Crit Care Nurs Q 21, $12\{25$.

[20] Jones B. F. 1998 A reappraisal of the use of infrared thermal image analysis in medicine, IEEE Trans Med Imaging 17, 1019\{1027.

[21] Shevelev I. A. 1998 Functional imaging of the brain by infrared radiation (thermoencephaloscopy), Progr Neurobiol 56, $269\{305$.

[22] Ford R. G. and Ford K. T. 1997 Thermography in the diagnosis of headache: Seminars in neurology, Semin Neurol 17, $343\{349$.

[23] Sterns E. E., Zee B., SenGupta S., and Saunders F. W. 1996 Thermography: Its relation to patho- logic characteristics, vascularity, proliferation rate, and survival of patients with invasive ductal carci- noma of the breast, Cancer Interdisc Int J Am Can- cer Soc 77, $1324\{1328$.

[24] Niboyet J. 1958 Nouvelle constatations sur les proprietes electriques des ponts Chinois, Bull Soc Acup 30, 7.

[25] Darras J.-C., Vernejoul P. D., and Albarede P. 1992 Nuclear medicine and acupuncture: a study on the migration of radioactive tracers after injection at acupoints, Am J Acupunct 20, $245\{256$.
[26] Nakatani Y. 1956 An aspect of the study of Ryodoraku, Clinic Chin Med 3, 54.

[27] Chen K.-G. 1996 Applying quantum interference to EDST medicine testing, IEEE Eng Med Biol Mag 15, $64\{66$.

[28] Yeap K., Voon C., Hiraguri T., and Nisar H. 2019 A compact dual-band implantable antenna for medical telemetry, Microwave Opt Technol Lett 61, $2105\{2109$. [29] Oh Z. X., Yeap K. H., Voon C. S., Lai K. C., and Teh P. C. 2020 A multiband antenna for biomedical telemetry and treatments, J Phys Conf Ser, 1502, 012013.

\section{Creative Commons Attribution License 4.0 (Attribution 4.0 International, CC BY 4.0)}

This article is published under the terms of the Creative Commons Attribution License 4.0

https://creativecommons.org/licenses/by/4.0/deed.en_US

Yassine SABRI was born on Octobre 28th, 1984 in Rabat city, Rabat province, Morocco. She received her Bachelor of Science (B.Sc), in field of Science and Technology, from the Mohamed 5 University of Rabat, Morrocco, in 2006. She completed her Master of Science (Msc), infield of Engineering, from the Universiti Couaib Douakaly in 2008 and then joined the Laboratory of Science and Technology, laysia to continue his study to Doctor of Technology (PhD) in field of WSN Technology. In 2013, she joined the Department of science and Technology, ISGA Rabat, as an assistant professor. his research interests include Wireless Sensor Networks, Evolutionary Computation, Internet of Things (IoT) and Mobile Computing. In recent years, she has focused on processing of Cloud Computing, Big Data, Articial Intelligence, Machine Learning evolutionary algorithms, software framework. She has collaborated actively with researchers in several other disciplines of science and technology.

Siham Aouad State Doctorate in Computer Engineering from EMI, 2014, she works in the fields of research Optimization of self-organization protocols for wireless sensor networks, Securing the Internet of Objects and the Cloud; Virtualization of SDN and NFV Networks. She is a teacher-researcher for the subject of Systems and Network Administration; IT security ; Virtualization Cloud computing;

Abderrahim Maizate currently works at the Depart- ment of Computing Science, Hassan II University of Casablanca. His research interests include fields such as Wireless communications, WSN, smart cities, NDN, AI, Virtualization, cloud computing and security. 